\title{
Three-dimensional speckle tracking echocardiography in evaluating left ventricular function in patients with triple vessel coronary artery disease without myocardial infarction
}

\author{
Xiuxiu Cui ${ }^{1}$, Huaying Bo ${ }^{1}$, Yu Dong ${ }^{1}$, Ying Wang ${ }^{1}$, ying li $^{1}$, wenxing chang $^{1}$, Tingting $\mathrm{Yu}^{1}$, \\ and Guangsen $\mathrm{Li}^{1}$ \\ ${ }^{1}$ Department of Ultrasound the Second Affiliated Hospital of Dalian Medical University \\ Dalian China
}

November 6, 2021

\begin{abstract}
Purpose Using three-dimensional speckle tracking echocardiography (3D-STE) to evaluate left ventricular (LV) function in patients with triple vessel coronary artery disease(TVD) without myocardial infarction. Methods Sixty patients with TVD without myocardial infarction were divided into two groups according to the results of coronary angiography. Group B $(\mathrm{n}=31): 50 \%[?]$ the stenosis rates of all triple vessel coronary artery; $75 \%$; Group $\mathrm{C}(\mathrm{n}=29)$ :the stenosis rates of all triple vessel coronary artery[?]75\%. Thirty healthy subjects were recruited as the group A. We measured LV end-diastolic and end-systolic volume (LVEDV, LVESV) and LV ejection fraction (LVEF) using real-time three-dimensional echocardiography. The 3D-STE parameters of LV included global longitudinal strain (GLS), global area strain (GAS), global radial strain (GRS) and global circumferential strain (GCS). Results In group C, LVEDV and LVESV were significantly increased (all $\left.P_{j} 0.05\right)$, while LVEF, GLS, GRS, GCS and GAS were significantly decreased compared with groups A and B (all $P$ ¡0.05). In groups A and B, there were no statistical differences in LVEDV, LVESV and LVEF. However, GLS, GCS and GAS were lower in group B than in group A (all $P$ i 0.05$)$. Conclusion Our study shows that 3D-STE can evaluate the LV function in patients with triple vessel coronary artery disease without myocardial infarction through multiple strain parameters.
\end{abstract}

\section{Introduction}

Coronary heart disease (CHD) has been a hot spot in cardiovascular disease research. It is a heart disease caused by the formation of coronary atherosclerotic plaque, which result in stenosis or obstruction of the vascular lumen, leading to myocardial ischemia, hypoxia and necrosis. According to the report published in 2016, CHD accounts for $20 \%$ of deaths in Europe, and is still one of the diseases with the highest morbidity and mortality in the world ${ }^{[1,2]}$. CHD involving three vessels disease (TVD) is a more extensive and serious disease than common CHD, and the change of left ventricular (LV) function was earlier than that of structure. The main function of the LV is to transport blood to all parts of the body and undertake the blood supply to the whole body. So the effective evaluation of LV function in patients with TVD is very meaningful to the treatment and prognosis of patients. Traditional echocardiography has become the first choice for clinicians to diagnose heart disease, but it is less sensitive in early identification of cardiac function changes ${ }^{[3]}$. The three-dimensional speckle tracking echocardiography (3D-STE) is a quantitative tool to evaluate LV function with high accuracy and reliability ${ }^{[4]}$. Nesser et al. ${ }^{[5]}$ found that 3D-STE in assessment of LV systolic function had a remarkable correlation with cardiac MRI. 3D-STE can provide more cardiac function evaluation indexes for clinical and detect LV subclinical myocardial injury early ${ }^{[6-8]}$. This study used 3D-STE to quantitatively analyze the changes of systolic LV strain parameters and evaluate LV function in patients with TVD without myocardial infarction. 


\section{Methods}

\section{Study population and grouping principles}

Our study enrolled patients, who had been clinically diagnosed with CHD at the department of cardiology, from hospital admissions made between June 2019 and December 2020 in our hospital. Our diagnostic criteria for CHD was consistent with the diagnostic and therapeutic guidelines of CHD from the European Society of Cardiology (ESC) published in $2013^{[9]}$. Among them, sixty patients with TVD were selected as the experimental group. Diagnostic criteria of TVD was as follows: coronary angiography showed that the diameter stenosis rates of three subepicardial vessels (left anterior descending coronary artery, circumflex coronary artery, right coronary artery) and / or their main branches were all[?]50\%. 60 patients with TVD were divided into two groups: 31 patients with the stenosis rate of all triple vessel coronary arteryi $75 \%$ served as group B (age, $45^{\sim} 77$ years; mean age, $57.41 \pm 8.29$ years; ratio of females to males: 20:11), 29 patients with all three-vessel stenosis rate[?] $75 \%$ served as group $C$ (age, $44^{\sim} 71$ years; mean age, 57.37+-8.16 years; ratio of females to males: 20:9). At the same time, we recruited 30 gender and age-matched healthy subjects (age, $41^{\sim} 69$ years; mean age, $55.43+-7.54$ years; ratio of females to males: 18:12) as group A. And all of the above participants were in sinus rhythm.

Exclusion criteria included: 1) therapeutic history of coronary revascularization; 2) myocardial infarction; 3) cardiac injury caused by coronary heart disease-related complications, such as papillary muscle rupture, ventricular wall rupture, interventricular septal perforation; 4) cardiac enlargement and cardiac dysfunction resulted by other types of heart disease, such as dilated cardiomyopathy, congenital heart disease, rheumatic heart disease, chronic pulmonary heart disease, valvular disease; 5) combined with hypertension, diabetes, nephrotic syndrome that contribute to heart damage. We also excluded the poor quality images.

Before the examination, we recorded the basic information of all participants on the checklist including name, gender, age, height and weight, then calculated body mass index (BMI, $\mathrm{kg} / \mathrm{m} 2$ ) and body surface area $\left(\mathrm{BSA}, \mathrm{m}^{2}\right)$. After taking a $10 \mathrm{~min}$ break, a physician who was blind the purpose of the study measured the blood pressure (BP) and the heart rate (HR) of all participants. And $4 \mathrm{~mL}$ venous blood samples were retrieved in fasting in the morning from three groups of participants, and the serum was obtained by centrifuging venous blood at $3000 \mathrm{r} / \mathrm{min}$ for $10 \mathrm{~min}$ and stored in the refrigerator(-30). Then the content of N-terminal pro-brain natriuretic peptide (NT-proBNP) of the serum were measure by photochemical method. LV end-diastolic pressure (LVEDP) was measured by left cardiac catheterization after coronary angiography in groups B and C, and the acquisition of ultrasonic images should be carried out within two days after left cardiac catheterization.

\section{Conventional echocardiographic measurements}

In our study, a GE Vivid E9 doppler ultrasound instrument equipped with an M5S-D probe (1.5-4.5MHz) was introduced to obtain 2-dimensional images. The ultrasound examination was conducted when the subjects lay in the left lateral decubitus position and keep steady breathing with connecting a three-lead electrocardiography (ECG). Three groups of participants completed an assessment of echocardiography from multiple views including the LV long-axis, apical 4-chamber and apical 2-chamber. We measured the conventional parameters which included interventricular septal thickness and LV posterior wall thickness at end-diastolic (IVSTd and PWTd), LV end-diastolic diameter (LVDd) and LV end-systolic diameter (LVDs), transmitral peak early and late inflow diastolic velocity (E and A), early-diastolic tissue velocity of septal and lateral mitral annulus (Sept e' and Lat e'), then computed respectively E/A and E/mean e'.

\section{Real-time three-dimensional echocardiography and 3D-STE measurements}

To acquire 3D echocardiographic LV full volume images, we used a matrix-array 4V-D probe (1.7-3.3 MHz). In real-time dynamic three-dimensional pattern, the frame rates of echocardiographic image were 25-50 frames/s. When the entire LV myocardium, endocardium, and epicardium were clearly displayed in apical 4-chamber view, we told the patients to hold their breath after a calm breath in order to eliminate breathing motion artifacts. Furthermore, to make sure a clear image, it is necessary to adjust the buttons that control 
the depth and width of the probe during the operation. Then turning into $4 \mathrm{D}$ full volume pattern, we continued to collect real-time dynamic three-dimensional images of LV in four consecutive cardiac cycles which can be analyzed with 3D-STE. At last, starting 4-dimensional automatic LV quantitative analysis software (4D-Auto LVQ) to analyze all the collected full-volume images. This software will automatically delineate the edocardial boundary of the LV, which can be manually adjusted if required. The parameters including LV end-diastolic volume (LVEDV), LV end-systolic volume (LVESV), LV ejection fraction (LVEF) were measured and strain curves for 17 segments of the LV wall were obtained. Global longitudinal strain (GLS), global area strain (GAS), global radial strain (GRS) and global circumferential strain (GCS) of 17 myocardial segments in each direction were got by computing the weighted average of the peak systolic strain values. The left atrial intima was traced in the same way to measure the left atrial maximum volume, and calculated the left atrial maximum volume index (LAVImax, LAVImax=LAVmax/BAS). Each parameter was measured three times by a physician, and the average value was taken.

\section{Statistical analysis}

We analyzed all data using SPSS 19.0 software (SPSS 19.0, Inc., Chicago, IL, USA). All continuous variables conforming to normal distribution were showed as mean values +- standard deviations. Comparisons of the gender among the three groups were made by using $\chi^{2}$-test. Differences of three groups were compared by analysis of one-way ANOVA. LSD-t test was used to compare the differences between two groups. For analyzing the correlations of NT-proBNP and LVEDP with 3D-STE parameters and in three groups, we used Pearson linear correlation analysis. The values of $\mathrm{P} j 0.05$ demonstrated that the results were statistically significance.

\section{Results}

\section{Clinical characteristics and NT-proBNP}

There was no obvious difference among the three groups of participants in the age, gender, BMI, BP and HR (all $\left.\mathrm{P}_{\dot{\varepsilon}} 0.05\right)$. In terms of level of NT-proBNP, group $\mathrm{C}$ was the highest among three groups, and group $\mathrm{B}$ had higher NT-proBNP level than that in group A (all $\left.\mathrm{P}_{j} 0.05\right)$. LVEDP was increased in groups B and $\mathrm{C}$, and LVEDP was increased in group $\mathrm{C}$ compared with group $\mathrm{B}(\mathrm{P}<0.05)$.(Shown in Table 1)

\section{Conventional echocardiographic parameters}

No difference was showed in IVSTd and PWTd among three groups (both $\mathrm{P}_{\mathrm{j}} 0.05$ ). In group $\mathrm{C}, \mathrm{E} / \mathrm{mean}$ e', LVDs and LVDd were increased (all $\mathrm{P}_{j} 0.05$ ), while E/A, LVEF, Sept e' and Lat e' were decreased (all $\left.\mathrm{P}_{\mathrm{j}} 0.05\right)$ compared to groups A and B. In group B, we found that E/mean e' was increased, while Sept e' and Lat e' were decreased compared with group A (all $\mathrm{P}_{j} 0.05$ ). There were no obvious difference in terms

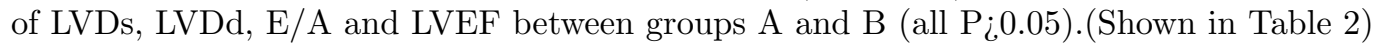

\section{D and 3D-STEparameters}

Compared with groups B and A, LVEDV and LVESV were obviously increased (all $\mathrm{P}_{j} 0.05$ ), whereas LVEF was obviously declined in group $\mathrm{C}\left(\mathrm{P}_{j} 0.05\right)$. The parameters of LVEDV, LVESV and LVEF were no obvious differences in groups $\mathrm{A}$ and $\mathrm{B}$ (all $\mathrm{P} \dot{\mathrm{i}} 0.05$ ). The patients in group $\mathrm{C}$ had higher LAVImax compared to groups A and B, and LAVImax level for group B was higher than for group A. GLS, GRS, GCS, GAS in group C were obviously lower (all $\mathrm{P}_{j} 0.05$ ) than those in groups A and B. GLS, GCS and GAS in group B were lower than those in group A (all $\left.\mathrm{P}_{\mathfrak{i}} 0.05\right)$ and GRS was also reduced, but there was no difference $\left(\mathrm{P}_{\dot{\iota}} 0.05\right)$. (Shown in Table 3 and Figure 1)

\section{Correlation analysis}

In order to make the results more intuitive, we used the absolute values instead of negative value of threedimensional strain parameters in the Pearson linear correlation. GLS, GRS, GCS and GAS were negatively related to NT-proBNP in group $\mathrm{C}(\mathrm{r}=-0.866 、 \mathrm{r}=-0.587 、 \mathrm{r}=-0.428 、 \mathrm{r}=-0.600 、 \mathrm{P}<0.001 、 \mathrm{P}=0.001 、 \mathrm{P}$ $=0.020 、 \mathrm{P}=0.010)$. And GLS is the only parameter that demonstrated a negative relationship with NTproBNP in group $\mathrm{B}(\mathrm{r}=-0.642 、 \mathrm{P}<0.010)$. And correlation analysis showed that the absolute values of 
three-dimensional strains were negatively correlated with LVEDP in groups B and C: GLS ( $\mathrm{r}_{\mathrm{B}}=-0.664, \mathrm{P}$ $\left.<0.001 ; \mathrm{r}_{\mathrm{C}}=-0.832, \mathrm{P}<0.001\right)$, GRS $\left(\mathrm{r}_{\mathrm{B}}=-0.449, \mathrm{P}=0.011 ; \mathrm{r}_{\mathrm{C}}=-0.668, \mathrm{P}<0.001\right)$, GCS $\left(\mathrm{r}_{\mathrm{B}}=-0.390, \mathrm{P}=\right.$ $\left.0.030 ; \mathrm{r}_{\mathrm{C}}=-0.484, \mathrm{P}=0.008\right)$ and $\mathrm{GAS}\left(\mathrm{r}_{\mathrm{B}}=-0.468, \mathrm{P}=0.008 ; \mathrm{r}_{\mathrm{C}}=-0.586, \mathrm{P}=0.001\right)$.

\section{Discussion}

As a subtype of CHD, TVD is more serious than single-vessel and double-vessel disease, which has a higher incidence of cardiovascular events and mortality ${ }^{[10]}$. At present, we need a technique to evaluate the changes of LV function early and accurately. Conventional echocardiography considers the LVEF is the most common parameter to evaluate myocardial systolic function ${ }^{[11]}$, but relevant studies have shown that the sensitivity of it is poor in detecting mild myocardial dysfunction ${ }^{[12]}$. Speckle tracking is a new echocardiographic technique developed in recent years. At present, 2D-STE has been widely used in clinical and experimental research $^{[13,14]}$. But the limitation of 2D-STE is that the tracking of myocardial acoustic spots is limited to the two-dimensional section, which makes it untraceable when some of the spots move completely outside the twodimensional section. The 3D-STE overcomes the'out-of-plane'phenomenon of 2D-STE in scanning ${ }^{[15,16]}$. It can quantitatively evaluate the deformation of regional myocardium in multiple directions, and reflect motion and function of myocardium in three-dimensional space more realistically and accurately ${ }^{[17,18]}$. Crosby et al. ${ }^{[19]}$ study on local myocardial function using speckle tracking technology shows that 3D-STE can accurately identify the myocardium with local dysfunction.

In our study, the LV structure of the group B was no obvious difference, and LVEF was also within the normal range compared with group $\mathrm{A}$. However, the group $\mathrm{C}$ had larger LV volume and lower LVEF than those in other two groups. The LV structure and LVEF can remain normal in group B, because the motion of myocardium in the ischemic region may be compensated by adjacent normal myocardium. With the aggravation of the degree of stenosis, the range of affected myocardium expands, and the LV function is gradually decompensated, resulting in cardiac enlargement and heart failure.

The myocardium of the LV is divided into three layers: the inner layer of the right hand spiral, the middle layer of the ring spiral and the outer layer of the left hand spiral ${ }^{[20,21]}$. Due to the orientations of myocardial fibers are different, its movement directions are also different. When the inner layer and outer layer myocardial fibers contract, there is movement in the direction of the long axis, and when the middle layer myocardium contracts, there is movement in the direction of the short axis. GLS, GCS and GRS respectively represent the myocardial deformation in the longitudinal, circumferential and perpendicular directions of endocardium. In addition, GAS is a new parameter ${ }^{[22,23]}$, which is the integration of GLS and GCS, and it represents the area change rate of endocardium. Longitudinal motion of myocardium is mainly determined by the myocardium fibers in the inner layer, and circumferential are determined by the myocardium fibers in the middle layer ${ }^{[24,25]}$.

Our study found that the GLS in groups B and C were obviously reduced compared to control group, and the changes were statistically significant. Blood supply to the endocardium comes from the vessels at the end of the coronary artery and studies have shown that change of GLS is dominated by the endocardial myocardium $^{[26]}$. So the endocardium is more sensitive to ischemia and hypoxia when the patients with TVD have myocardial ischemia, which means that GLS has changed under normal conditions of LVEF ${ }^{[27]}$. If stenosis rate of TVD further aggravates leading to the overload of cardiac and the enlargement of cardiac, the decrease of GLS is more significant. In our study, it was found that the GCS in groups B and C were obviously lower than that in control group $(\mathrm{P} ; 0.05)$. The circumferential contraction of the short axis of the cardiac is mainly caused by the movement of the middle layer. Generally, myocardial ischemia gradually expands from the endocardium to the epicardium ${ }^{[28]}$, so when myocardial ischemia and hypoxia occur, the middle layer of myocardial segments are affected, resulting in reduction of GCS. As a specific indicator of 3D-STE, GAS is the integration of GLS of the ventricular long axis and GCS of the ventricular short axis, so the significant reduction of GAS can better assess the cardiac systolic dysfunction in patients with TVD.

GRS represents the thickness of the ventricular wall and the change of the LV volume from the LV short axis view ${ }^{[29]}$. In our study, the reduction of GRS in groups B were not statistically significant compared 
with group A. It might be because the radial thickening of ventricular wall is the result of the interaction of cross fibers between different myocardial layers and the inner and outer of fibers perpendicular to fiber orientation. Under normal physiological conditions, the systolic thickening ability of ventricular wall mainly depends on the myocardial fibers in the intimal layer. When the contractility of endocardial myocardium is reduced due to ischemia, the outer subepicardial layer contributes to thickening and short-axis function through cross-fiber shortening. In the group C, GRS was obviously decreased compared with the other two groups. It showed that the further development of the disease had affected the middle layer, which was consistent with the research results of Winter et al ${ }^{[30]}$. In addition, the research of Chan et al ${ }^{[24]}$. showed that when the degree of coronary stenosis is severe or even myocardial infarction occurs, the peak value of radial strain can show a significant decrease. Therefore, GRS is less sensitive to early identification of myocardial ischemia of TVD.

Diastolic movement of the heart is an active energy consuming process, and the threshold of diastolic function reduction is lower than systolic function ${ }^{[31]}$. Therefore, the reduction of diastolic function is earlier than systolic function during myocardial ischemia. Our study evaluated LV diastolic function in line with the guideline issued by the American society of echocardiography in $2016^{[32]}$, which proved that the LV diastolic function was decreased in groups B and C, and group C was lower than group B. This is consistent with the results of Rydberg et al. ${ }^{[33]}$, indicating that $\mathrm{LV}$ diastolic function is closely related to the degree of coronary artery stenosis. LVEDP measured by left cardiac catheterization is the gold standard for the diagnosis of LV diastolic dysfunction ${ }^{[34]}$. This study found that LVEDP in groups $\mathrm{B}$ and $\mathrm{C}$ increased, and that in group $\mathrm{C}$ was higher than in group B, the difference was statistically significant. At present, there are few studies on the correlation between 3D-STE and LVEDP, and only shin et al. ${ }^{[35]}$ research confirmed that GLS and GAS were closely related to LVEDP. We results also suggest that the GLS and GAS had significant correlations with LVEDP in group B and group C. In addition, this study found that GCS and GRS also had high correlations with LVEDP in group B and group C. Therefore, 3D-STE has a certain reference value for evaluating LV diastolic function in the patients with TVD.

NT-proBNP is produced by the hydrolysis of proBNP which can produce BNP at the time, and NT-proBNP may be more specific to cardiac activity than BNP ${ }^{[36]}$. Studies have confirmed BNP may be actively degraded in peripheral blood, so the test of NT-proBNP is more reliable ${ }^{[37]}$. NT-proBNP, as a polypeptide neurohormone, is mainly synthesized and secreted by ventricular myocytes ${ }^{[38]}$. In recent years, more and more attention has been paid to the diagnostic and prognostic of BNP in patients with different clinical types of CHD ${ }^{[39-41]}$. Some studies have showed the change of serum NT-proBNP closely related to myocardial ischemia and hypoxia. When the systolic function of cardiac is abnormal, the ventricular volume load increases and the ventricular wall is pulled, causing the synthesis and release of NT-proBNP ${ }^{[42]}$. Our results showed the level of NT-proBNP was distinctly difference among the three groups. NT-proBNP of group $\mathrm{C}$ was highest among of three groups. It was due to the severe stenosis of the TVD result in ischemia of cardiomyocytes and LV systolic dysfunction, which stimulates the synthesized and secreted of NT-proBNP by ventricular myocytes. In group C, the study demonstrated each 3D-STE strain parameter were negatively correlated with NT-proBNP. And the correlation analysis also demonstrated GLS was negatively correlated with NT-proBNP in group B. Therefore, the level of serum NT-proBNP is consistent with the 3D-STE in the evaluation of LV systolic function in patients with TVD.

\section{Study limitations}

The present study still existed some limitations. First of all, 3D-STE uses low frame rate to collect real-time three-dimensional full volume images for analysis, which will cause the loss of myocardial motion information and low image resolution. In addition, the image quality is very high. It needs to show the endocardium clearly and then trace it. If necessary, it needs to be manually adjusted, which exists great subjectivity. Furthermore, 3D-STE is not suitable for image acquisition of patients with irregular heart rhythm, and the scanning angle of 3D-STE is narrow, so it is difficult to obtain complete image information for patients with large heart. Finally, we had a relatively small study population, so it is necessary to expand the sample size for further study in the future. 


\section{Conclusions}

All in all, LV function is closely related to the stenosis degree of TVD. In the early stage of TVD, when the overall left ventricular systolic function is normal, the local left ventricular systolic function has been damaged. As a new technique, 3D-STE can detect the changes of myocardial function earlier in patients with TVD and quantitatively evaluate the global LV function through multiple global strain parameters. This may provide a more effective method for monitoring the change of LV systolic strain and evaluating LV systolic function of TVD without myocardial infarction.

\section{Reference}

1. Townsend N, Wilson L, Bhatnagar P, et al. Cardiovascular disease in Europe: epidemiological update 2016[J]. Eur Heart J, 2016, 37: 3232-3245

2. Benjamin EJ, Blaha MJ, Chiuve SE, et al. Heart Disease and Stroke Statistics-2017 Update: A Report From the American Heart Association[J]. Circulation, 2017, 135: e146-e603

3. Shimoni S, Gendelman G, Ayzenberg O, et al. Differential effects of coronary artery stenosis on myocardial function: the value of myocardial strain analysis for the detection of coronary artery disease $[\mathrm{J}]$. J Am Soc Echocardiogr, 2011, 24: 748-757

4. Wang D, Zhang L, Zeng Q, et al. Assessment of left ventricular performance in heart transplant recipients by three-dimensional speckle tracking imaging[J]. Medicine (Baltimore), 2017, 96: e8129

5. Nesser HJ, Mor-Avi V, Gorissen W, et al. Quantification of left ventricular volumes using threedimensional echocardiographic speckle tracking: comparison with MRI[J]. Eur Heart J, 2009, 30: 1565-1573

6. Zhou Q, Shen J, Liu Y, et al. Assessment of left ventricular systolic function in patients with iron deficiency anemia by three-dimensional speckle-tracking echocardiography[J]. Anatol J Cardiol, 2017, 18: 194-199

7. Voilliot D, Huttin O, Hammache N, et al. Impact of Global and Segmental Hypertrophy on TwoDimensional Strain Derived from Three-Dimensional Echocardiography in Hypertrophic Cardiomyopathy: Comparison with Healthy Subjects[J]. J Am Soc Echocardiogr, 2015, 28: 1093-1102

8. Li L, Zhang PY, Ran H, et al. Evaluation of left ventricular myocardial mechanics by three-dimensional speckle tracking echocardiography in the patients with different graded coronary artery stenosis $[\mathrm{J}]$. Int $\mathrm{J}$ Cardiovasc Imaging, 2017, 33: 1513-1520

9. Montalescot G, Sechtem U, Achenbach S, et al. 2013 ESC guidelines on the management of stable coronary artery disease: the Task Force on the management of stable coronary artery disease of the European Society of Cardiology[J]. Eur Heart J, 2013, 34: 2949-3003

10. Ryu KS, Park HW, Park SH, et al. Comparison of clinical outcomes between culprit vessel only and multivessel percutaneous coronary intervention for ST-segment elevation myocardial infarction patients with multivessel coronary diseases[J]. J Geriatr Cardiol, 2015, 12: 208-217

11. Solomon SD, Anavekar N, Skali H, et al. Influence of ejection fraction on cardiovascular outcomes in a broad spectrum of heart failure patients[J]. Circulation, 2005, 112: 3738-3744

12. Yip GW, Zhang Q, Xie JM, et al. Resting global and regional left ventricular contractility in patients with heart failure and normal ejection fraction: insights from speckle-tracking echocardiography $[\mathrm{J}]$. Heart, 2011, 97: 287-294

13. Langeland S, Wouters PF, Claus P, et al. Experimental assessment of a new research tool for the estimation of two-dimensional myocardial strain[J]. Ultrasound Med Biol, 2006, 32: 1509-1513 
14. Leitman M, Lysyansky P, Sidenko S, et al. Two-dimensional strain-a novel software for real-time quantitative echocardiographic assessment of myocardial function[J]. J Am Soc Echocardiogr, 2004, 17: 1021-1029

15. Biswas M, Sudhakar S, Nanda NC, et al. Two- and three-dimensional speckle tracking echocardiography: clinical applications and future directions[J]. Echocardiography, 2013, 30: 88-105

16. Thebault C, Donal E, Bernard A, et al. Real-time three-dimensional speckle tracking echocardiography: a novel technique to quantify global left ventricular mechanical dyssynchrony[J]. Eur J Echocardiogr, 2011, 12: $26-32$

17. Hayat D, Kloeckner M, Nahum J, et al. Comparison of real-time three-dimensional speckle tracking to magnetic resonance imaging in patients with coronary heart disease[J]. Am J Cardiol, 2012, 109: 180-186

18. Tee M, Noble JA, Bluemke DA. Imaging techniques for cardiac strain and deformation: comparison of echocardiography, cardiac magnetic resonance and cardiac computed tomography[J]. Expert Rev Cardiovasc Ther, 2013, 11: 221-231

19. Crosby J, Amundsen BH, Hergum T, et al. 3-D speckle tracking for assessment of regional left ventricular function[J]. Ultrasound Med Biol, 2009, 35: 458-471

20. Nakatani S. Left ventricular rotation and twist: why should we learn?[J]. J Cardiovasc Ultrasound, 2011, 19: $1-6$

21. Goffinet C, Chenot F, Robert A, et al. Assessment of subendocardial vs. subepicardial left ventricular rotation and twist using two-dimensional speckle tracking echocardiography: comparison with tagged cardiac magnetic resonance[J]. Eur Heart J, 2009, 30: 608-617

22. Reant P, Barbot L, Touche C, et al. Evaluation of global left ventricular systolic function using threedimensional echocardiography speckle-tracking strain parameters[J]. J Am Soc Echocardiogr, 2012, 25: 68-79

23. Wang Q, Gao Y, Tan K, et al. Assessment of left ventricular function by three-dimensional speckletracking echocardiography in well-treated type 2 diabetes patients with or without hypertension[J]. J Clin Ultrasound, 2015, 43: 502-511

24. Chan J, Hanekom L, Wong C, et al. Differentiation of subendocardial and transmural infarction using two-dimensional strain rate imaging to assess short-axis and long-axis myocardial function[J]. J Am Coll Cardiol, 2006, 48: 2026-2033

25. Kim HK, Sohn DW, Lee SE, et al. Assessment of left ventricular rotation and torsion with twodimensional speckle tracking echocardiography $[\mathrm{J}]$. J Am Soc Echocardiogr, 2007, 20: 45-53

26. Perk G, Tunick PA, Kronzon I. Non-Doppler two-dimensional strain imaging by echocardiography-from technical considerations to clinical applications[J]. J Am Soc Echocardiogr, 2007, 20: 234-243

27. Kraigher-Krainer E, Shah AM, Gupta DK, et al. Impaired systolic function by strain imaging in heart failure with preserved ejection fraction[J]. J Am Coll Cardiol, 2014, 63: 447-456

28. Mizuguchi Y, Oishi Y, Miyoshi H, et al. The functional role of longitudinal, circumferential, and radial myocardial deformation for regulating the early impairment of left ventricular contraction and relaxation in patients with cardiovascular risk factors: a study with two-dimensional strain imaging $[\mathrm{J}]$. J Am Soc Echocardiogr, 2008, 21: 1138-1144

29. Nakai H, Takeuchi M, Nishikage T, et al. Effect of aging on twist-displacement loop by 2-dimensional speckle tracking imaging[J]. J Am Soc Echocardiogr, 2006, 19: 880-885

30. Winter R, Jussila R, Nowak J, et al. Speckle tracking echocardiography is a sensitive tool for the detection of myocardial ischemia: a pilot study from the catheterization laboratory during percutaneous coronary intervention[J]. J Am Soc Echocardiogr, 2007, 20: 974-981 
31. Kane GC, Karon BL, Mahoney DW, et al. Progression of left ventricular diastolic dysfunction and risk of heart failure[J]. JAMA, 2011, 306: 856-863

32. Nagueh SF, Smiseth OA, Appleton CP, et al. Recommendations for the Evaluation of Left Ventricular Diastolic Function by Echocardiography: An Update from the American Society of Echocardiography and the European Association of Cardiovascular Imaging[J]. J Am Soc Echocardiogr, 2016, 29: 277-314

33. Rydberg E, Willenheimer R, Erhardt L. The prevalence of impaired left ventricular diastolic filling is related to the extent of coronary atherosclerosis in patients with stable coronary artery disease[J]. Coron Artery Dis, 2002, 13: 1-7

34. Fan JL, Su B, Zhao X, et al. Correlation of left atrial strain with left ventricular end-diastolic pressure in patients with normal left ventricular ejection fraction[J]. Int J Cardiovasc Imaging, 2020, 36: 1659-1666

35. Shin SH, Park SD, Woo SI, et al. Utility of global strain by two-dimensional and three-dimensional speckle tracking for assessing left ventricular diastolic function: comparison with pressure wire analysis[J]. Korean Circ J, 2013, 43: 615-621

36. Valli N, Gobinet A, Bordenave L. Review of 10 years of the clinical use of brain natriuretic peptide in cardiology[J]. J Lab Clin Med, 1999, 134: 437-444

37. Goetze JP, Jensen G, Moller S, et al. BNP and N-terminal proBNP are both extracted in the normal kidney[J]. Eur J Clin Invest, 2006, 36: 8-15

38. Hosoda K, Nakao K, Mukoyama M, et al. Expression of brain natriuretic peptide gene in human heart. Production in the ventricle[J]. Hypertension, 1991, 17: 1152-1155

39. Wei G, Yaqi R, Ningfu W, et al. N-terminal prohormone B-type natriuretic peptide and cardiovascular risk in stable coronary artery disease: a meta-analysis of nine prospective studies[J]. Rev Cardiovasc Med, 2013, 14: e92-98

40. Eliasdottir SB, Klemenzson G, Torfason B, et al. Brain natriuretic peptide is a good predictor for outcome in cardiac surgery[J]. Acta Anaesthesiol Scand, 2008, 52: 182-187

41. Balta S, Demir M, Demirkol S, et al. Plasma B-type natriuretic peptide level in patients with coronary artery bypass surgery[J]. Kaohsiung J Med Sci, 2013, 29: 648-649

42. Chen TH, Lin CL, Shih JJ, et al. Plasma B-type natriuretic peptide in predicting outcomes of elective coronary artery bypass surgery[J]. Kaohsiung J Med Sci, 2013, 29: 254-258 

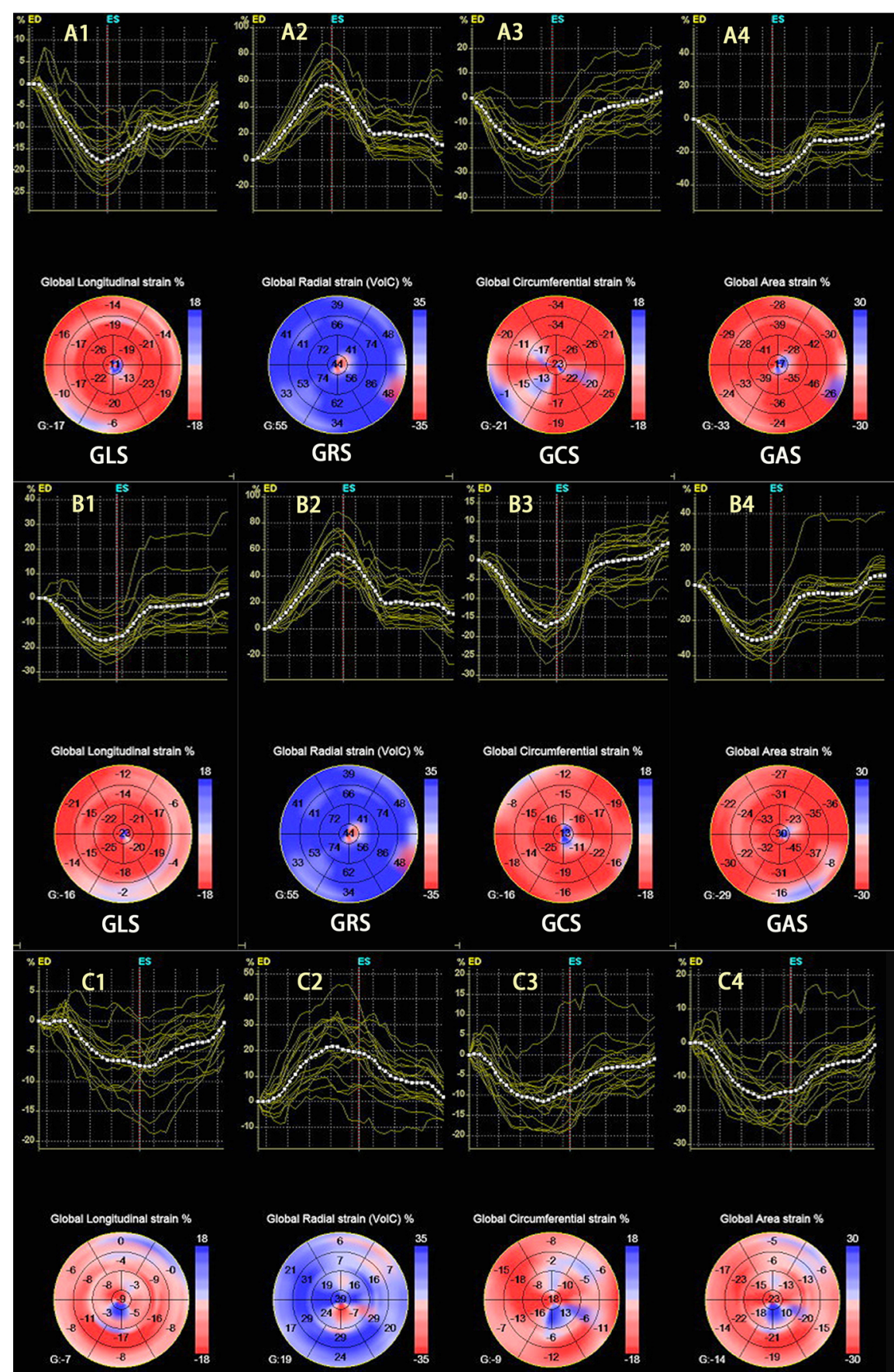

GRS

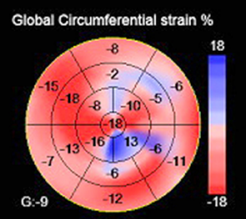

GCS

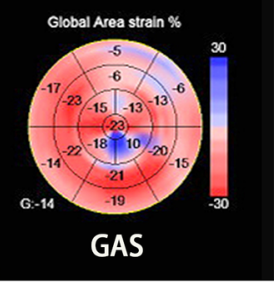

\section{Hosted file}

Legend for Figures.docx available at https://authorea.com/users/398665/articles/544431-threedimensional-speckle-tracking-echocardiography-in-evaluating-left-ventricular-functionin-patients-with-triple-vessel-coronary-artery-disease-without-myocardial-infarction

Hosted file 
Table 1_2.docx available at https://authorea.com/users/398665/articles/544431-threedimensional-speckle-tracking-echocardiography-in-evaluating-left-ventricular-functionin-patients-with-triple-vessel-coronary-artery-disease-without-myocardial-infarction

\section{Hosted file}

Table 1_1.docx available at https://authorea.com/users/398665/articles/544431-threedimensional-speckle-tracking-echocardiography-in-evaluating-left-ventricular-functionin-patients-with-triple-vessel-coronary-artery-disease-without-myocardial-infarction

\section{Hosted file}

Table2 . docx available at https : //authorea. com/users/398665/articles/544431-three-dimensionalspeckle-tracking-echocardiography-in-evaluating-left-ventricular-function-in-patientswith-triple-vessel-coronary-artery-disease-without-myocardial-infarction

\section{Hosted file}

Table3. docx available at https : //authorea. com/users/398665/articles/544431-three-dimensionalspeckle-tracking-echocardiography-in-evaluating-left-ventricular-function-in-patientswith-triple-vessel-coronary-artery-disease-without-myocardial-infarction 